\title{
ON INJURIES TO PERIPHERAL NERVES AND THEIR SURGICAL TREATMENT.
}

By FRANK C. PURSER, M.D., Major, R.A.M.C.; Physician to Mercer's Hospital, Dublin.

[Read in the Section of Surgery, April 7, 1916.]

A PROBLEM that is frequently before us, and one that is very perplexing, is that of deciding on the condition of some injured peripheral nerve and advising as to its treatment. It is the question on which I am asked for an opinion perhaps more often than in any other; and I acknowledge that almost every case present,s some difficulty. No case requires more than these to be judged on its own merits and not by any rule of thumb. And after a most conscientious examination and consideration of the merits, subsequent developments often prove one to have been a bad prophet. Cases in which operation has been confidently recommended have improved surprisingly before it was undertaken, and others in which physicotherapy and time seemed all that was needed to effect perfect recovery, have consumed much of both with but disappointing results. This experience is pretty general. The difficulties lie largely in the number of the factorsmany of them incalculable-which have to be taken into consideration; and to some extent in the examination of the patients, many of whom, to say the least of it, do not over-exert themselves in helping the examiner. By this communication to-night on the treatment of injured peripheral nerves I hope to start discussion, and to hear the 
experience of others. I shall deal only with those aspects of the question which may be expected to interest the surgeon and physician alike.

In the first place, certain considerations are necessary in examining these cases.

When a mixed nerve is cut across there follows, of course, as a result, paralysis of sensation and of motion in the skin area and muscles supplied by that nerve. When a nerve is not wholly divided, but only partially, there are at least modifications of normal sensation and motion. Now, as a correct judgment of the condition and progress of an injured nerve depends on a just estimate of its loss of function, and especially on loss of sensibility in the area it supplies, I must delay a little in considering these preliminaries. Loss of sensation is two-fold; it is a loss in the cutaneous area and loss in the deeper structures, muscles, tendons, \&c., supplied by the divided nerve. These two sorts of sensibility, the cutaneous or superficial and the deep, are distinct from one another. I emphasise this point, though I know it is well recognised theoretically, for I think that it is not so well recognised in practice, with the result that one reads some otherwise inexplicable accounts of very rapid return of nerve function after operation.

Cutaneous, or superficial, sensibility comprises the recognition of light touches as such and of their correct Jocalisation. It allows one to recognise as separate two points when simu!laneously applied-what has come to be known as the "compass" test. It comprises also the recognition of the painful sharpness of a pin-prick and the discrimination of teimperatures even when tiey differ from one another by only a few degrees. Chief reliance is to be placed on the modifications of cutaneous sensibility. 
Deep sensibility is less easily judged, and even in very complete injuries does not seem always to be absent. As far as our present enquiry goes, it concerns the recognition of pressure as a "touch" more or less heavy on the skin and subjacent structures, and a sense of pain when the pressure is severe. It will be readily understood from this that care must be taken in testing cutaneous sensibility not to stimulate deep sensibility. In testing the cutaneous sense of touch, only cotton wool must be used, or something equally light. Many use a finger tip, but no matter how delicately applied it is far too heavy. It causes deformity of the shape of the skin, and in so doing stimulates deep sensibility. Indeed, so much is the lightness of the object applied to be insisted on that I warn you that if you are meditating making a contribution to the study of sensation, it will not receive the respectful consideration of neurophysiologists unless the cotton wool used be of long staple like that used by jewellers. For a similar reason a wire pin, which is always blunt, should not be used for testing the sense of pain. Its application will often be acknowledged-but only as a deep touch-where a needle or a steel pin are not felt at all.

The local condition of the part to be tested must also be considered; it should be comfortably supported and pleasantly warm. And the patient himself should be comfortable and as willing as possible to give help. The man who wants his tea, or for some other reason resents your coming just when you do, will give much less accurate answers than he will give next day when the sun is shining and you are welcome. This reference to sunshine is not a mere pleasantry; there is no doubt that in any but crude testing a bright, fresh day conduces to answering quite appreciably more accurate than is obtainable on a damp, 
gloomy one. It is always wise to have as few spectators as possible.

When a mixed nerve, then, is divided in continuitye.g., the median at the elbow-the sensory loss is a failure to recognise pressure over some of the flexor muscles of the forearm, and a loss of sense of touch of cotton wool, of the painfulness of a pin-prick, and of temperature in an area corresponding pretty accurately to the cutaneous distribution of the nerve as given in anatomical text-books. Motor power will be lost in the muscles whose branches of supply are given off distal to the level of section.

But this statement is not exactly the truth. Those stimuli which can be increased in degree without altering their character-the stimuli of pain by pricking and of temperature are wholly lost only over an area considerably less than that of the anatomical distribution of the nerveso that, in the case of the median, as far as the palm is concerned, while all sense of touch is lost in the anatomical distribution of the nerve, recognition of severe pin-pricks and the extremer degrees of temperature are lost only in two fingers and a portion of the palm.

If the nerve is left divided the area where all sensibility is lost tends, for some reason, to shrink-and not as we might think, to enlarge; and on the motor side the muscles waste and undergo changes (that are of clinical value) in their reactions to electricity. Later, if care be not taken to prevent it, the opponents of the paralysed muscles will shorten and contractions will occur, and the tissues in the area supplied may undergo tropic changes, and any trifling injury be long in healing.

But if the divided nerve be promptly sutured, and the wound heals without suppuration, recovery of power and of sensibility may be expected. Motor power, and with 
it some return of electrical exciteability, may be hoped for in about nine months. Sensation is recovered more slowly. Between two and four months after suture the wholly anæsthetic area begins to shrink, and by the end of a year it is altogether responsive to pin-pricks and the extremer degrees of temperature. The lighter degrees of sensation-of touch, localisation and fine discrimination of temperature-are not so quick in returning. They take twelve to eighteen months. Last of all to return is the power of localising touches correctly and appreciating the simultaneous application of two points. And even when objectively sensation is wholly recovered there remains often for a year or two a subjective sense of " something different" in the affected hand-a numbness when stimulated, or a cold feeling.

All these times are only approximate, and vary greatly in different cases. Recovery depends cn many things besides the skill of the surgeon. The younger the patient and the nearer to the periphery the injury, and the more assiduous the post-operative treatment, the better the prognosis. Suppuration in the wound makes the outlook worse. An injury close to the spinal cord will recover slowly and imperfectly. And recovery depends on the nerve involved. In a nerve like the musculospiral it is better than in the ulnar, which supplies muscles of highly specialised action.

These shcrt remarks will suffice to show what may be expected, at best, in, say, a glass-cut median nerve which has been sutured within a few hours of being injured. Unfortunately a wholly complete recovery is not the rule under even the most favourable circumstances. Some weakness or noticeable sensory defect remains. Less still can it be expected in the injuries inflicted in war, where 
we have to deal with men who are often run-down in health, whose wounds are septic, whose muscles and nerves have been of necessity left unattended to, and who are suffering from bruises, lacerations, fractures, and other extra-neural complications. In these cases diagnosis and advice as to treatment are matters of particular difficulty ; they take time to be arrived at, and are rarely to be decided at a single examination.

Nerve injuries fall into two groups.

A group, numerically far the smaller, in which there is persistent total loss of function, and a group in which there is partial loss of function. A case in either group may be complicated by pain or by hyperæsthesia.

Total or partial loss of function may be due to complete or incomplete anatomical discontinuity of a nerve. Or they may be due to the pressure of exudate, hæmorrhage, fragment of a projectile, or of bone, \&c., without any discernible anatomical lesion at all-a physiological discontinuity, and either total or partial discontinuity, but especially the latter, may be associated with pain or with hyperwsthesia. Let us consider the case of total loss of function.

It must be remembered that often as the result of a violent blow from a projectile a whole limb may show both motor and sensory paralysis, and this without any discernible nerve lesion at all. This paralysis-ascribed to shock to the nerve-usually clears up rapidly and completely in a fortnight. One sees little of it at home. And, again, one must remember in making a diagnosis, that paralysis of a functional or hysterical nature often occur with, or independently of, organic lesions of the peripheral nerves. It is often difficult enough to detect these, but the fact that the sensory loss is generally of a "glove" or "sock" distribution and does not correspond to a cutaneous 
area of any nerve or nerves; that there are no vaso-motor or trophic changes, and that the muscles react readily to Faradism will help to settle the question. And a careful enquiry into the circumstances of the injury and paralysis will also help. I have at present under treatment a rather extreme case which illustrates these points : a man with a healed but formidable-looking scar about three inches below his right elbow joint. The scar surrounds about three-fifths of the forearm. The arm below the scar is very thin in comparison with the left arm, and there is total paralysis of the muscles supplied by the musculospiral and ulnar nerves, and almost complete paralysis of those supplied by the median. The man had been considered unfit for service on account of this paralysis of the arm, but on one case sheet there was a diagnosis of hysteria, and he was sent to me for further opinion. The muscles I found all reacted to Faradism readily. There was anæsthesia to touch and pain and temperature as high as the scar, and in the whole forearm, though certainly some cutaneous nerves must have escaped the original laceration. There was no vaso-motor change, and on making an enquiry into the history of the condition $I$ found the scar was due to a crush by a cask edge ten years before he had enlisted, which was eight years ago. He had been in Flanders several months, and had been sent home with a slight flesh wound in the contra-lateral shoulder. The paralysis came on suddenly on his receiving orders to join his regiment, orders which came at the same time as a domestic tragedy at home and news of the death of his brother in action abroad. I have seen several other cases where a shot, through the forearm resulted in quite unequivocal functional paralysis, motor and sensory, which had been accepted as organic, and reported accordingly. 
But having made sure that the total loss of function is organic one must decide what to do. There is no sign or symptom that will enable one to decide whether the nerve is severed or involved in inflammatory tissue. The proper treatment in such a case seems to me to be to record exactly the sensory loss and the motor loss with the electrical reactions, but especially the sensory loss. Improvement in sensibility will be recognised long before improvement in motility, and on the change of sensory symptoms one can make some estimate as to the condition of the nerve. Should there be no change in six weeks or two months I think the nerve should be exposed. If it be found divided it must be sutured; if only compressed it must be freed. The outlook in the two cases is different. No very great recovery can be hoped for in secondary suture, and what does come is often rather long in coming. But much more may be expected both in completeness and rapidity of recovery in a nerve that is only compressed in a scar.

Cases of complete nerve-division are very seldom associated with much pain. I have never seen such a case with any pain to speak of. But if it do occur it is in the same category as the nerves in a painful amputation stump, and should be dealt with similarly.

It is of the utmost importance that the injured part should be well cared for while awaiting developments, and after operation if that be undertaken. The essentials are that the limb should be rested and kept warm, and, most necessary of all, that the paralysed muscles should be kept constantly relaxed. Massage to these and passive movement of all joints to prevent contractures, and the application of any form of electricity to which the muscles may respond, are to be practised. If the muscles respond to no 
electric treatment its application is but waste of time. The effect of relaxation on paralysed muscles can sometimes be shown very well. I have seen cases of drop-wrist where no reasonable electrical stimulation gave any contraction in the extensors, till they had been relaxed by means of a splint for three or four days, when it was easy enough to induce one.

Now let us consider the much larger group of cases of partially divided nerves. In these cases symptoms vary from at most complete motor and sensory loss to no more than a subjective appreciation of abnormality. Often the motor loss seems, as far as one can compare the two, out of all proportion to the sensory loss. The sensory loss may be entirely overlooked in a superficial examination. But it is by it that one can judge more than by any other the progress of a case. The history of this injury will illustrate all these points. A man was hit by a bullet which fractured the humerus in the lower third and incidentally caused paralysis of the whole forearm and hand. The median and ulnar supplied areas rapidly recovered, but the muscles supplied by the musculospiral were obviously more deeply involved. The wound was a little septic at the time, and no operation was contemplatedthis was three months after the injury. I was asked to see him, and I gathered that sensation was considered to be normal. When his splint was removed he had drop-wrist, and it was not easy to get any electrical reactions at all. I tested sensation, and he never failed to acknowledge the stimulus, and he made no remarks on the sensation it produced. I was going to write sensation down as perfect, but first I asked him as to comparison between the two hands - and he said it was " quite different" from the left hand. I then tested him with the compasses and tested 
his power of localisation. Both were very defective. One month afterwards the voluntary motor power was as absent as before, the wound had healed, and prospects of having to operate were distantly contemplated. But I found that although sensation was still subjectively "quite different" from the left hand power of appreciating two points as such had greatly improved, and localisation was perfect. Six weeks later very fair voluntary power had returned in the extensors, and this is steadily increasing. (Charts were shown illustrating this and other sensory abnormalities).

The return of power and sensation in a partially divided nerve is due to the release of the nerve from the pressure of hæmorrhages or inflammatory exudation. It is not due to the downgrowths of proximal axis cylinders and their fortuitous linking up with the distal remains of the nerve. Once inflammation has come to an end the loss of function becomes stationary. The question then arises whether to operate or to leave alone. The general considerations which I have enumerated will guide here as in the case of a wholly divided nerve. No complete cure can be looked for, and sometimes things are made worse by operation. If contractures have formed it is waste of time operating. Speaking generally, in the majority of cases it will be found inadvisable to operate. (There is great luck in getting a good result, and the chance of making things worse is not negligible even with the most skilled. The patient will be advised not to risk a little power in his own hand for twice as much on the knees of the gods.)

But there are, I think, two very definite calls for operation. The first is where all inflammation has subsided and the patient is left with little or no power and fairly good sensibility. The risk of losing the sensibility may 
well be taken on even an offchance of getting back power. And another definite indication for operation is the presence of severe pain or of hyperæsthesia. Pain is a much more common complication in partial nerve division than it is in cases of total division, and it is of a particularly, in the lay sense, "unnerving" kind. To rid the victim of it it is well even to risk much motor loss. But the disabling factor of the pain must be weighed against the disablement of a paralysed nerve, and treatment on analgesic lines should be extensively tried before more radical measures. It might be well in some cases to consider the relief of pain by injecting alcohol into the sheath of a nerve - even of a mixed nerve. Its effects wear off in time, and by then perhaps the pain might be found to have worn off, too, and a fairly functioning nerve be left.

Apropos of pain and hyperæsthesia, it is necessary to say a word about trophic disturbances. In my experience they are not very great, but in cases where there is much pain, in these the trophic changes have been more marked. Any procedure which will leave the affected area a sense of pain on pricking and even a crude appreciation of temperature will probably protect it against any gross trophic changes.

I now come to discuss what may be done at operation. It is a matter I feel shy at introducing, and I would not have had the presumption to do so at all had it not been hinted to me that my doing so would not be taken amiss.

I have spoken of the difficulties of deciding whether to operate or not, and have pointed them out rather than solved them. The difficulties of technique in operating are outside my personal experience, but I see that they are very great, and I have endless admiration for the skill that overcomes them. 
There are some main guiding rules. One is that no operation should be undertaken unless the wound be healed, or at least aseptic. The chances of suturing giving any other result than a bulb of fibrous tissue would be none. Another rule is that a nerve must be handled with the extremity of gentleness. Indeed, it should never be caught up at all or held back on a hook, but liftèd by its sheath. This is to avoid injury to the delicate nerve elements themselves and also to avoid hæmorrhage into the nerve or its sheath, for any exudation will lead to subsequent development of obstructing fibrous tissue. I have seen it done, and often heard it stated that a man's leg may be lifted off the table by his exposed sciatic nervebut the best results seem to be got when force is perfected in gentleness. And this gentleness must be extended to the dissection of scar tissue from around a nerve to avoid the exudation that may lead to more scar tissue. Some surgeons claim that they have spent up to four hours in such dissecting, and found the results justified the time and care spent. In German reports I have seen it stated that this dissection may be greatly helped by the injection into the nerve sheath of normal saline solution. Finally, in suturing a nerve the most exact apposition of the nerve fibres in the divided ends must be aimed at. No other expedient is of any avail.

A question will arise in the numerous cases in which a spindle or bulb of fibrous tissue is found in the course of a nerve. Should it be removed or left? Neither eye nor finger can tell.how much nerve fibre or how little traversés it. The loss of function found by clinical examination must be borne in mind, and if it be thought reasonable that resection and secondary suture will give better results let it be done. But no recognisable intact bundle of nerve fibres should ever be severed. 
In resection the nerve should be cut with a razor. Scissors, be they ever so sharp, crush the nerve fibres, and crushing is the surest way to stop their growth. The nerve should be cut till the fibrils are plainly visible, and then the two ends must be brought into the exactest apposition and under as little tension as possible-and the whole must be as dry as possible and protected from exudation as much as possible by a sleeve of vein, Cargile membrane, or layers of fat.

When a nerve is ready for suture it may be that, despite all stretching and flexing of joints to relax it, the ends cannot be brought into apposition. The prognosis is bad then. Of all the "bridges" that have been made between the ends, none offer so good a chance of success as the insertion of a piece of sensory nerve from the patient himself. And no "bridge" of nerve over four inches in length can be expected to function. I have heard of much longer "bridges" being successfully laid. But four inches is on the long side. Perhaps next best is a " bridge" taken from an amputated limb. A nerve from a rabbit or other animal is useless. And there is no unequivocal evidence that guides like a portion of vein, decalcified bone, or strands of catgut ever lead to any recovery or continuity.

Should approximation of the ends be impossible, and bridging also fail, some result may be hoped for by grafting one nerve into another. This is done by suturing an end into a notch made in a sound nerve. This operation is common enough as regards the facial and spinal accessory nerves or the facial and hypoglossal, it is less common in the case of nerves in the limbs. It has been done, however, successfully in the case of the median and ulnar. The first fully successful case was one where the median 
was divided in its length and one portion joined with the peripheral end of the accidentally divided musculospiral. At the end of two years and three months there was good power in all the extensor muscles of the wrist and fingers, but not of the thumb.

In these cases of nerve anastomosis there must be a sorting out and re-arrangement for transmission of impulses in the spinal cord. At first, as in the case of the spinal accessory and facial anastomosis there is an associated movement, but in the course of time, as the cord accommodates itself, this becomes dissociated. A rather more recent form of anastomosis is the insertion into the trunk of a sound nerve of both the proximal and peripheral ends of the divided nerve. Here the spinal cord has to make no rearrangements. Impulses will pass to it and from it by the normal segmental roots. The main line of the uninjured nerve has double traffic over part of its length. I have seen no detailed accounts of this method, but preliminary reports are favourable.

In isolating a "flap" of sound nerve care must be taken not to take too much. Apparently one-quarter or onethird of the nerve can be divided without noticeable loss of function-it is this fact that makes nerve anastomosis possible at all. But a nerve must not be notched close to a branch, for there the fibres going to make up the branch are segregated into one bundle, and this bundle may be divided wholly in the raising of the flap.

I have, perhaps, not been as optimistic about the chances of surgery in these injuries as would be complimentary at a surgical meeting. This is not from want of appreciation of surgery, but it is from an appreciation of the very real difficulties the surgeon has to face. It is difficult enough to deal with the nerve when it is found; it is sometimes 
more difficult still to find it. I believe the possibilities of surgery are increasing in this field; but $I$ think if neuroplasty is to have the success of, say, abdominal surgery, that even the simplest operation will have to be looked on as of necessity a lengthy business, so that the greatest patience and care may be exercised in securing complete isolation and accurate apposition of the nerve-endings, with the minimum of tension and the minimum of handling. 